\title{
Pythagorean fuzzy topological spaces
}

\author{
Murat Olgun $^{1}$ (D) $\cdot$ Mehmet Ünver $^{1}$ (1) $\cdot$ Şeyhmus Yardımcı ${ }^{1}$
}

Received: 13 November 2018 / Accepted: 12 February 2019 / Published online: 6 March 2019

(c) The Author(s) 2019

\begin{abstract}
In the present paper, we introduce the notion of Pythagorean fuzzy topological space by motivating from the notion of fuzzy topological space. We define Pythagorean fuzzy continuity of a function defined between Pythagorean fuzzy topological spaces and we characterize this concept. Using the concept of continuity, we also give a method to construct a Pythagorean fuzzy topology on a given non-empty set.
\end{abstract}

Keywords Pythagorean fuzzy subset $\cdot$ Pythagorean fuzzy topological space $\cdot$ Pythagorean fuzzy continuity

Mathematics Subject Classification 03E72 · 94D05 · 54C05

\section{Introduction}

Classical topology has been progressed by taking its motivation from classical analysis and it has many applications on several areas of research such as machine learning, data analysis, data mining, and quantum gravity [1-7]. Besides, the notion of topology refers the connection between spatial objects and features and it can be used to describe some certain spatial functions and to conceive data sets that have better quality control and greater data integrity. Homeomorphisms that are the isomorphisms of the category of topological spaces play an important role in the theory. If there exists a homeomorphism among two topological spaces, then these spaces have exactly the same topological properties. The topological part of a homeomorphism is the notion of continuity [1]. Moreover, continuity explains the term of closeness in the mathematical models. In this context, continuity is one of the most important properties of a function defined between two topological spaces.

In 1968, Chang [8] defined the concept of fuzzy topological space and generalized some basic notions of topology such as open set, closed set, continuity and compactness to fuzzy topological spaces. Following this study, Lowen gave

Murat Olgun

olgun@ankara.edu.tr

1 Ankara University, Ankara, Turkey another definition of a fuzzy topological space by changing a basic property of topology $[9,10]$. Besides, in 1995, Coker introduced the notion of intuitionistic fuzzy topological space and studied some analogue versions of some concepts of classical topology such as continuity and compactness [11]. Further results on intuitionistic fuzzy topological spaces can be found in [12-14]. Some authors were also concerned with fuzzy metric spaces (see, e.g. [15]). Thus, degree-theoretic structure of fuzziness has been considered in the notion of topology instead of certain fixed boundaries. Furthermore, some authors studied the concept of fuzzy soft topological space and its applications in decision-making environment $[16,17]$.

The main purpose of this paper is to extend the notions of fuzzy topological space and intuitionistic fuzzy topological space by introducing the notion of Pythagorean fuzzy topological space. We define the concept of Pythagorean fuzzy topological space by following the idea of Chang [8]. After defining Pythagorean fuzzy topological spaces, we investigate the continuity of a function between two Pythagorean fuzzy topological spaces which we call Pythagorean fuzzy continuity. For this purpose, we define the notions of image and the pre-image of a Pythagorean fuzzy subset with respect to a function and we investigate some basic properties of these notions. We also construct the coarsest Pythagorean fuzzy topology on a non-empty set $X$ which makes a given function $f: X \rightarrow Y$ Pythagorean fuzzy continuous where $Y$ is a Pythagorean fuzzy topological space. In this context, the results obtain in the present paper can be used to get 
more realistic results in the areas of research such as decision making, data analysis and machine learning. In the future works, categorical properties of Pythagorean fuzzy topological spaces, applications of Pythagorean fuzzy topological spaces on decision making theory and Pythagorean fuzzy soft topological spaces may be studied.

\section{Basic concepts on Pythagorean fuzzy subsets}

In 1965, Zadeh generalized the standard notion of set by defining the notion of fuzzy set [18] that has many applications on economy, business, decision making, data mining, etc. A fuzzy subset $A$ of a given set $X$ is defined with a function $\mu_{A}: X \rightarrow[0,1]$ which is called the membership function of $A[18,19]$. For a given $x \in X$, the value $\mu_{A}(x)$ is said to be the degree or grade of membership of $x$ to $A$. Besides, the grade of non-membership of $x$ to $A$ is given with $1-\mu_{A}(x)$. This idea obviously generalizes the idea of standard set theory. Indeed, in the standard set theory the membership function of a set $A$ can be considered as the characteristic function of $A$ which is equal to one if $x \in A$ and zero otherwise. The notion of fuzzy subset has been generalized to some more general notions of nonstandard fuzzy subset. For instance, an intuitionistic fuzzy subset $A$ of $X$ is the pair $\left(\mu_{A}, v_{A}\right)$ of a membership function $\mu_{A}: X \rightarrow[0,1]$ and a non-membership function $v_{A}: X \rightarrow[0,1]$ with $\mu_{A}(x)+v_{A}(x) \leq 1$ for any $x \in X$ [20]. Another example of a non-standard fuzzy subset is the interval-valued fuzzy subsets which was studied in depth by Mendel [21,22]. On the other hand, in 2014, Yager [23] introduced the notion of Pythagorean fuzzy subset which is a new class of non-standard fuzzy subsets and which has many effective applications in natural and social sciences (see, e.g. [24-40]).

A Pythagorean fuzzy subset $A$ of a non-empty set $X$ is a pair $\left(\mu_{A}, v_{A}\right)$ of a membership function $\mu_{A}: X \rightarrow[0,1]$ and a non-membership function $v_{A}: X \rightarrow[0,1]$ with $\mu_{A}^{2}(x)+v_{A}^{2}=r_{A}^{2}(x)$ for any $x \in X$ where $r_{A}: X \rightarrow[0,1]$ is a function which is called the strength of commitment at point $x$ [23]. The notion of the Pythagorean fuzzy subset has a basic geometric mean: Let $\left(r_{A}(x), \theta_{A}(x)\right)$ be the polar coordinates of $\left(\mu_{A}(x), v_{A}(x)\right)$ for a point $x \in X$. If we define $d_{A}(x):=\left(1-\theta_{A}(x)\right) \frac{\pi}{2}$, then the function $d_{A}: X \rightarrow[0,1]$ can be considered the direction of commitment at point $x$ [23]. It is obvious that the function $d_{A}$ scales the first quadrant between zero and one, i.e. if $\theta_{A}(x)=\frac{\pi}{2}$ then $\mu_{A}(x)=0$ and $v_{A}(x)=r_{A}(x)$ which means the direction $d_{A}(x)=0$ and if $\theta_{A}(x)=0$ then $\mu_{A}(x)=r_{A}(x)$ and $v_{A}(x)=0$ which means the direction $d_{A}(x)=1$. Therefore, a Pythagorean fuzzy subset $A$ can be expressed by either $\left(\mu_{A}, v_{A}\right)$ or $\left(r_{A}, d_{A}\right)$. Note here that Pythagorean fuzzy subsets can be still appli-

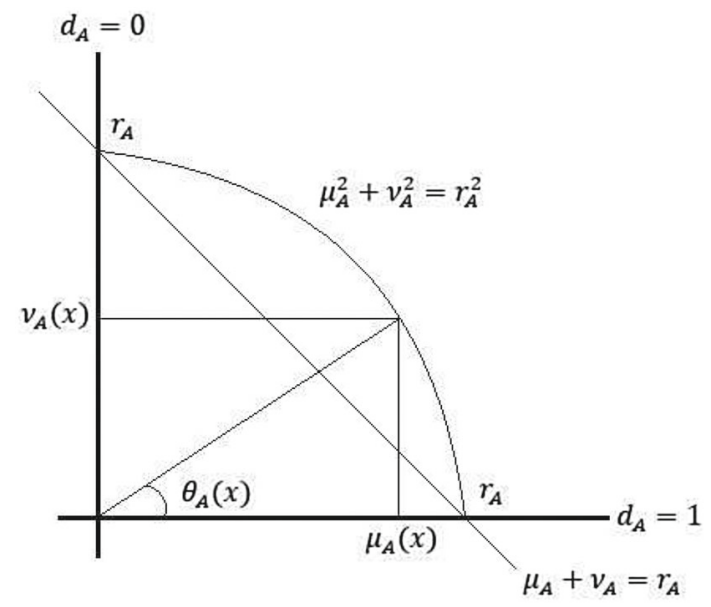

Fig. 1 Comparison of intuitionistic fuzzy subsets and Pythagorean fuzzy subsets

cable whenever one cannot use intuitionistic fuzzy subsets. Therefore, Pythagorean fuzzy subsets are more effective than intuitionistic fuzzy subsets as well as fuzzy subsets (see Fig. 1).

Now let us recall the set operations over Pythagorean fuzzy subsets [23]:

Definition 1 Let $A=\left(\mu_{A}, v_{A}\right)$ and $B=\left(\mu_{B}, v_{B}\right)$ be two Pythagorean fuzzy subsets of a set $X$. Then,

(i) the complement of $A$ is defined by $A^{c}:=\left(v_{A}, \mu_{A}\right)$,

(ii) the intersection of $A$ and $B$ is defined by $A \cap B:=$ $\left(\min \left\{\mu_{A}, \mu_{B}\right\}, \max \left\{v_{A}, v_{B}\right\}\right)$,

(iii) the union of $A$ and $B$ is defined by $A \cup B=$ $\left(\max \left\{\mu_{A}, \mu_{B}\right\}, \min \left\{v_{A}, v_{B}\right\}\right)$,

(iv) we say $A$ is a subset of $B$ or $B$ contains $A$ and we write $A \subset B$ or $B \supset A$ if $\mu_{A} \leq \mu_{B}$ and $v_{A} \geq v_{B}$.

Note here that, if the union and the intersection are infinite, then we use supremum "sup" and infimum "inf" instead of maximum "max" and minimum "min", respectively.

Throughout this paper, we use the notation $1_{X}$ for the Pythagorean fuzzy subset $(1,0)$ and we use the notation $0_{X}$ for the Pythagorean fuzzy subset $(0,1)$, i.e.

$\mu_{1_{X}}=1$ and $v_{1_{X}}=0$

and

$\mu_{0_{X}}=0$ and $v_{0_{X}}=1$.

\section{Topological spaces}

In this section, we define the concept of Pythagorean fuzzy topological space. We also study the continuity of a function defined among Pythagorean fuzzy topological spaces. 
Definition 2 Let $X \neq \varnothing$ be a set and let $\tau$ be a family of Pythagorean fuzzy subsets of $X$. If

(T1) $1_{X}, 0_{X} \in \tau$,

(T2) for any $A_{1}, A_{2} \in \tau$, we have $A_{1} \cap A_{2} \in \tau$,

(T3) for any $\left\{A_{i}\right\}_{i \in I} \subset \tau$, we have $\bigcup_{i \in I} A_{i} \in \tau$ where $I$ is an arbitrary index set then $\tau$ is called a Pythagorean fuzzy topology on $X$.

In this case the pair $(X, \tau)$ is said to be a Pythagorean fuzzy topological space. Each member of $\tau$ is called an open Pythagorean fuzzy subset. The complement of an open Pythagorean fuzzy subset is called a closed Pythagorean fuzzy subset. As classical topologies or fuzzy topological spaces, the family $\left\{1_{X}, 0_{X}\right\}$ is called the indiscreet Pythagorean fuzzy topological space and the topology that contains all Pythagorean fuzzy subsets is called the discrete Pythagorean fuzzy topological space. Besides, a Pythagorean fuzzy topology $\tau_{1}$ on a set is said to be coarser than a Pythagorean fuzzy topology $\tau_{2}$ on the same set if $\tau_{1} \subset \tau_{2}$.

Following is an example of a Pythagorean fuzzy topological space:

Example 1 Let $X=\{1,2\}$. Consider the following family of Pythagorean fuzzy subsets $\tau=\left\{1_{X}, 0_{X}, A_{1}, \ldots, A_{5}\right\}$ where

$$
\begin{array}{llll}
\mu_{1}(1)=0.5 & v_{1}(1)=0.7 & \mu_{2}(1)=0.6 & v_{2}(1)=0.5 \\
\mu_{1}(2)=0.2 & v_{1}(2)=0.4 & \mu_{2}(2)=0.3 & v_{2}(2)=0.9 \\
\mu_{3}(1)=0.4 & v_{3}(1)=0.8 & \mu_{4}(1)=0.6 & v_{4}(1)=0.5 \\
\mu_{3}(2)=0.1 & v_{3}(2)=0.95 & \mu_{4}(2)=0.3 & v_{4}(2)=0.4 \\
\mu_{5}(1)=0.5 & v_{5}(1)=0.7 & \mu_{5}(2)=0.2 & v_{5}(2)=0.9
\end{array}
$$

and $\mu_{i}$ and $v_{i}$ are corresponding membership and nonmembership functions of $A_{i}$ for each $i=1, \ldots, 5$, respectively. Observe that $(X, \tau)$ is a Pythagorean fuzzy topological space.

As any fuzzy subset or intuitionistic fuzzy subset of a set can be considered as a Pythagorean fuzzy subset, we observe that any fuzzy topological space or intuitionistic fuzzy topological space is a Pythagorean fuzzy topological space as well. On the other hand, it is obvious that a Pythagorean fuzzy topological space needs not to be a fuzzy topological space or intuitionistic fuzzy topological space. Even an open Pythagorean fuzzy subset may be neither a fuzzy subset nor an intuitionistic fuzzy subset (see Example 1). The categorical relationship between these spaces may be studied in future works.

The notion of the neighbourhood of a point has an important place in the classical topology as many concepts such as continuity, closure and convergence are defined or characterized with the help of this notion. Chang gave the definition of a neighbourhood of a fuzzy open subset instead of a neigh- bourhood of a point [8]. Following the idea of Chang, we give the following definition:

Definition 3 Let $A, U$ be two Pythagorean fuzzy subsets in a Pythagorean fuzzy topological space. Then, $U$ is said to be a neighbourhood of $A$ if there exists an open Pythagorean fuzzy subset $E$ such that $A \subset E \subset U$.

The following fact is easy to prove:

Proposition 1 A Pythagorean fuzzy subset $A$ is open in a Pythagorean fuzzy topological space if and only if it contains a neighbourhood of its each subset.

Now, we give some new definitions to generalize some well-known ordinary topological results:

Definition 4 Let $X$ and $Y$ be two non-empty sets, let $f$ : $X \rightarrow Y$ be a function and let $A$ and $B$ be Pythagorean fuzzy subsets of $X$ and $Y$, respectively. Then, the membership and non-membership functions of image of $A$ with respect to $f$ that is denoted by $f[A]$ are defined by

$\mu_{f[A]}(y):= \begin{cases}\sup _{z \in f^{-1}(y)} \mu_{A}(z), & \text { if } f^{-1}(y) \text { is non-empty } \\ 0 & , \text { otherwise }\end{cases}$

and

$v_{f[A]}(y):= \begin{cases}\inf _{z \in f^{-1}(y)} v_{A}(z), & \text { if } f^{-1}(y) \text { is non-empty } \\ 1 & , \text { otherwise }\end{cases}$

respectively. The membership and non-membership functions of pre-image of $B$ with respect to $f$ that is denoted by $f^{-1}[B]$ are defined by

$\mu_{f^{-1}[B]}(x):=\mu_{B}(f(x))$

and

$v_{f^{-1}[B]}(x):=v_{B}(f(x))$,

respectively.

Note that $f[A]$ and $f^{-1}[B]$ are Pythagorean fuzzy subsets. In fact, since $\mu_{A}$ and $\nu_{A}$ are non-negative functions one can obtain

$$
\begin{aligned}
\mu_{f[A]}^{2}(y)+v_{f[A]}^{2}(y) & =\left(\sup _{z \in f^{-1}(y)} \mu_{A}(z)\right)^{2}+\left(\inf _{z \in f^{-1}(y)} v_{A}(z)\right)^{2} \\
& =\sup _{z \in f^{-1}(y)} \mu_{A}^{2}(z)+\inf _{z \in f^{-1}(y)} v_{A}^{2}(z)
\end{aligned}
$$




$$
\begin{aligned}
& =\sup _{z \in f^{-1}(y)}\left(r_{A}^{2}(z)-v_{A}^{2}(z)\right)+\inf _{z \in f^{-1}(y)} v_{A}^{2}(z) \\
& \leq \sup _{z \in f^{-1}(y)}\left(1-v_{A}^{2}(z)\right)+\inf _{z \in f^{-1}(y)} v_{A}^{2}(z)=1,
\end{aligned}
$$

whenever $f^{-1}(y)$ is non-empty. On the other hand if $f^{-1}(y)=\varnothing$, then we have

$\mu_{f[A]}^{2}(y)+v_{f(A)}^{2}(y)=1$.

The proof is trivial for $f^{-1}[B]$.

The following proposition gives some basic properties of image and pre-image:

Proposition 2 Let $X$ and $Y$ be two non-empty sets and let $f: X \rightarrow Y$ be a function. Then, we have

(i) $f^{-1}\left[B^{c}\right]=f^{-1}[B]^{c}$ for any Pythagorean fuzzy subset $B$ of $Y$

(ii) $f[A]^{c} \subset f\left[A^{c}\right]$ for any Pythagorean fuzzy subset $A$ of $X$.

(iii) if $B_{1} \subset B_{2}$ then $f^{-1}\left[B_{1}\right] \subset f^{-1}\left[B_{2}\right]$ where $B_{1}$ and $B_{2}$ are Pythagorean fuzzy subsets of $Y$.

(iv) if $A_{1} \subset A_{2}$, then $f\left[A_{1}\right] \subset f\left[A_{2}\right]$ where $A_{1}$ and $A_{2}$ are Pythagorean fuzzy subsets of $X$.

(v) $f\left[f^{-1}[B]\right] \subset B$ for any Pythagorean fuzzy subset $B$ of $Y$.

(vi) $A \subset f^{-1}[f[A]]$ for any Pythagorean fuzzy subset $A$ of $X$.

Proof (i) For any $x \in X$ and for any Pythagorean fuzzy subset $B$ of $Y$ we get from the definition of the complement that

$$
\begin{aligned}
\mu_{f^{-1}\left[B^{c}\right]}(x) & =\mu_{B^{c}}(f(x)) \\
& =v_{B}(f(x)) \\
& =v_{f^{-1}[B]}(x) \\
& =\mu_{f^{-1}[B]^{c}}(x) .
\end{aligned}
$$

Similarly one can have $v_{f^{-1}\left[B^{c}\right]}(x)=v_{f^{-1}[B]^{c}}(x)$. Therefore, we have $f^{-1}\left[B^{c}\right]=f^{-1}[B]^{c}$.

(ii) For any $y \in Y$ such that $f(y) \neq \varnothing$ and for any Pythagorean fuzzy subset $A$ of $X$, we can write

$$
\begin{aligned}
r_{f[A]}^{2}(y) & =\mu_{f[A]}^{2}(y)+v_{f(A)}^{2}(y) \\
& =\sup _{z \in f^{-1}(y)} \mu_{A}^{2}(z)+\inf _{z \in f^{-1}(y)} v_{A}^{2}(z) \\
& =\sup _{z \in f^{-1}(y)}\left(r_{A}^{2}(z)-v_{A}^{2}(z)\right)+\inf _{z \in f^{-1}(y)} v_{A}^{2}(z) \\
& \leq \sup _{z \in f^{-1}(y)} r_{A}^{2}(z)-\inf _{z \in f^{-1}(y)} v_{A}^{2}(z)+\inf _{z \in f^{-1}(y)} v_{A}^{2}(z) \\
& =\sup _{z \in f^{-1}(y)} r_{A}^{2}(z) .
\end{aligned}
$$

Now from (3.1), we have

$$
\begin{aligned}
\mu_{f\left[A^{c}\right]}(y) & =\sup _{z \in f^{-1}(y)} \mu_{A^{c}}(z) \\
& =\sup _{z \in f^{-1}(y)} v_{A}(z) \\
& =\sup _{z \in f^{-1}(y)} \sqrt{r_{A}^{2}(z)-\mu_{A}^{2}(z)} \\
& \geq \sqrt{\sup _{z \in f^{-1}(y)} r_{A}^{2}(z)-\sup _{z \in f^{-1}(y)} \mu_{A}^{2}(z)} \\
& \geq \sqrt{r_{f[A]}^{2}(y)-\mu_{f[A]}^{2}(y)} \\
& =v_{f[A]}(y) \\
& =\mu_{f[A]^{c}(y) .}
\end{aligned}
$$

The proof is trivial for each $y \in Y$ such that $f(y)=\varnothing$. On the other hand, we have $v_{f\left[A^{c}\right]}(y) \leq v_{f[A]^{c}}(y)$ using the same idea. Hence, we obtain $f[A]^{c} \subset f\left[A^{c}\right]$.

(iii) Assume that $B_{1} \subset B_{2}$. Then, we have for any $x \in X$ that

$$
\begin{aligned}
\mu_{f^{-1}\left[B_{1}\right]}(x) & =\mu_{B_{1}}(f(x)) \\
& \leq \mu_{B_{2}}(f(x)) \\
& =\mu_{f^{-1}\left[B_{2}\right]}(x) .
\end{aligned}
$$

Therefore, one can get $\mu_{f^{-1}\left[B_{1}\right]} \leq \mu_{f^{-1}\left[B_{2}\right]}$. Similarly, it is not difficult to show that $v_{f^{-1}\left[B_{1}\right]} \geq v_{f^{-1}\left[B_{2}\right]}$.

(iv) Assume that $A_{1} \subset A_{2}$ and let $y \in Y$. If $f(y)=\varnothing$, then the proof is trivial. Assume that $f(y) \neq \varnothing$. Then, we have

$$
\begin{aligned}
\mu_{f\left[A_{1}\right]}(y) & =\sup _{z \in f^{-1}(y)} \mu_{A_{1}}(z) \\
& \leq \sup _{z \in f^{-1}(y)} \mu_{A_{2}}(z) \\
& =\mu_{f\left[A_{2}\right]}(y) .
\end{aligned}
$$

Thus, $\mu_{f\left[A_{1}\right]} \leq \mu_{f\left[A_{2}\right]}$ follows. Similarly, we have $v_{f\left[A_{1}\right]} \geq v_{f\left[A_{2}\right]}$.

(v) For any $y \in Y$ such that $f(y) \neq \varnothing$, we can write

$$
\begin{aligned}
\mu_{f\left[f^{-1}[B]\right]}(y) & =\sup _{z \in f^{-1}(y)} \mu_{f^{-1}[B]}(z) \\
& =\sup _{z \in f^{-1}(y)} \mu_{B}(f(z)) \\
& =\mu_{B}(y) .
\end{aligned}
$$

On the other hand if $f(y)=\varnothing$, then we have $\mu_{f\left[f^{-1}[B]\right]}(y)=0 \leq \mu_{B}(y)$. Similarly, we have $v_{f\left[f^{-1}[B]\right]} \geq v_{B}$. 
(vi) For any $x \in X$, we have

$$
\begin{aligned}
\mu_{f^{-1}[f[A]]}(x) & =\mu_{f[A]}(f(x)) \\
& =\sup _{z \in f^{-1}(f(x))} \mu_{A}(z) \\
& \geq \mu_{A}(x) .
\end{aligned}
$$

Similarly, we have $v_{f^{-1}[f[A]]} \leq v_{A}$.

The concept of continuity is one of the well-known and important concepts of classical topology as it reformulates the notion of closeness in terms of open sets. We now give the definition of Pythagorean fuzzy continuity of a function.

Definition 5 Let $\left(X, \tau_{1}\right)$ and $\left(Y, \tau_{2}\right)$ be two Pythagorean fuzzy topological spaces and let $f: X \rightarrow Y$ be a function. Then, $f$ is said to be Pythagorean fuzzy continuous if for any Pythagorean fuzzy subset $A$ of $X$ and for any neighbourhood $V$ of $f[A]$ there exists a neighbourhood $U$ of $A$ such that $f[U] \subset V$.

The following theorem that characterizes the Pythagorean fuzzy continuity is a consequence of Proposition 2:

Theorem 1 Let $\left(X, \tau_{1}\right)$ and $\left(Y, \tau_{2}\right)$ be two Pythagorean fuzzy topological spaces and let $f: X \rightarrow Y$ be a function. Then, the following statements are equivalent:

(i) $f$ is Pythagorean fuzzy continuous.

(ii) For any Pythagorean fuzzy subset $A$ of $X$ and for any neighbourhood $V$ of $f[A]$, there exists a neighbourhood $U$ of $A$ such that for any $B \subset U$ we have $f[B] \subset V$.

(iii) For any Pythagorean fuzzy subset $A$ of $X$ and for any neighbourhood $V$ of $f[A]$, there exists a neighbourhood $U$ of $A$ such that $U \subset f^{-1}[V]$.

(iv) For any Pythagorean fuzzy subset $A$ of $X$ and for any neighbourhood $V$ of $f[A], f^{-1}[V]$ is a neighbourhood of $A$.

Proof (i) $\Rightarrow$ (ii) Assume that $f$ is Pythagorean fuzzy continuous, let $A$ be a Pythagorean fuzzy subset of $X$ and let $V$ be a neighbourhood of $f[A]$. Then, there exists a neighbourhood $U$ of $A$ such that $f[U] \subset V$. Now, if $B \subset U$, then we get $f[B] \subset f[U] \subset V$.

(ii) $\Rightarrow$ (iii) Assume that (ii) holds, let $A$ be a Pythagorean fuzzy set of $X$ and let $V$ be a neighbourhood of $f[A]$. From (ii), there exists a neighbourhood $U$ of $A$ such that for any $B \subset U$ we have $f[B] \subset V$. Then, we can write $B \subset$ $f^{-}[f[B]] \subset f^{-1}[V]$. As $B$ is an arbitrary subset of $U$, we have $U \subset f^{-1}[V]$.

(iii) $\Rightarrow$ (iv) Assume that (iii) holds. Let $A$ be a Pythagorean fuzzy subset of $X$ and let $V$ be a neighbourhood of $f[A]$.
Then from (iii), there exists a neighbourhood $U$ of $A$ such that $U \subset f^{-1}[V]$. Since $U$ is a neighbourhood of $A$ there exists an open Pythagorean fuzzy subset $P$ of $X$ such that $A \subset$ $P \subset U$. On the other hand as $U \subset f^{-1}[V]$, one can get $A \subset$ $P \subset f^{-1}[V]$ which implies $f^{-1}[V]$ is a neighbourhood of $A$.

(iv) $\Rightarrow$ (i) Assume that (iv) holds, let $A$ be a Pythagorean fuzzy subset of $X$ and let $V$ be a neighbourhood of $f[A]$. From the hypothesis, we have $f^{-1}[V]$ is a neighbourhood of $A$. Therefore, there exists an open Pythagorean fuzzy subset $P$ of $X$ such that $A \subset P \subset f^{-1}[V]$ which implies $f[P] \subset$ $f\left[f^{-}[V]\right] \subset V$. Moreover, as $P$ is open it is a neighbourhood of $A$. Hence, $f$ is Pythagorean fuzzy continuous.

Following is a characterization of Pythagorean fuzzy continuity. Note that similar result is one of the well-known results of classical topology.

Theorem 2 Let $\left(X, \tau_{1}\right)$ and $\left(Y, \tau_{2}\right)$ be two Pythagorean fuzzy topological spaces. A function $f: X \rightarrow Y$ is Pythagorean fuzzy continuous if and only if for each open Pythagorean fuzzy subset $B$ of $Y$ we have $f^{-1}[B]$ is an open Pythagorean fuzzy subset of $X$.

Proof Assume that $f$ is continuous. Let $B$ be an open Pythagorean fuzzy subset of $Y$ and let $A \subset f^{-1}[B]$. Then, we get $f[A] \subset B$. Since $B$ is open now from Proposition 1 there exists a neighbourhood $V$ of $f[A]$ such that $V \subset B$. Thus, Pythagorean fuzzy continuity of $f$ and (iv) of Theorem 1 imply that $f^{-1}[V]$ is a neighbourhood of $A$. On the other hand from (iii) of Proposition 2 we have $f^{-1}[V] \subset f^{-1}[B]$. Therefore, $f^{-1}[B]$ is a neighbourhood of $A$ as well. As $A$ is an arbitrary subset of $f^{-1}[B]$, from Proposition 1 the Pythagorean fuzzy subset $f^{-1}[B]$ is open.

Conversely, let $A$ be a Pythagorean fuzzy subset of $X$ and let $V$ be a neighbourhood of $f[A]$. Then, there exists an open Pythagorean fuzzy subset $P$ of $X$ such that $f[A] \subset P \subset$ $V$. Now, from the hypothesis $f^{-1}[P]$ is open. On the other hand, we can write $A \subset f^{-1}[f[A]] \subset f^{-1}[P] \subset f^{-1}[V]$. Hence, $f^{-1}[V]$ is a neighbourhood of $A$ which proves the Pythagorean fuzzy continuity of $f$.

We can obtain a Pythagorean fuzzy topology on a set $X$ when we are given a Pythagorean fuzzy topological space $Y$ and a function from $X$ to $Y$. We prove this fact with the following theorem:

Theorem 3 Let $X \neq \varnothing$ be a set, let $(Y, \tau)$ be a Pythagorean fuzzy topological space and let $f: X \rightarrow Y$ be a function. Then, there exists a coarsest Pythagorean fuzzy topology $\tau^{*}$ over $X$ such that $f$ is Pythagorean fuzzy continuous.

Proof Let us define a class of Pythagorean fuzzy subsets $\tau^{*}$ of $X$ by

$\tau^{*}:=\left\{f^{-1}[V]: V \in \tau\right\}$. 
We prove that $\tau^{*}$ is the coarsest Pythagorean fuzzy topology over $X$ such that $f$ is continuous.

(T1) We can write for any $x \in X$ that

$$
\begin{aligned}
\mu_{f^{-1}\left[0_{Y}\right]}(x) & =\mu_{0_{Y}}(f(x)) \\
& =0 \\
& =\mu_{0_{X}}(x)
\end{aligned}
$$

Similarly, we immediately have $v_{f^{-1}\left[0_{Y}\right]}(x)=v_{0_{X}}(x)$ for any $x \in X$ which implies $f^{-1}\left[0_{Y}\right]=0_{X}$. Now, as $0_{Y} \in \tau$ we have $0_{X}=f^{-1}\left[0_{Y}\right] \in \tau^{*}$. In like manner, it is easy to see that $1_{X}=f^{-1}\left[1_{Y}\right] \in \tau^{*}$.

(T2) Assume that $V_{1}, V_{2} \in \tau^{*}$. Then, for $i=1,2$ there exists $B_{i} \in \tau$ such that $f^{-1}\left[B_{i}\right]=V_{i}$ which implies $\mu_{f^{-1}\left[B_{i}\right]}=\mu_{V_{i}}$ and $v_{f^{-1}\left[B_{i}\right]}=v_{V_{i}}$. Thus, we obtain for any $x \in X$ that

$$
\begin{aligned}
\mu_{V_{1} \cap V_{2}}(x) & =\min _{i=1,2} \mu_{V_{i}}(x) \\
& =\min _{i=1,2} \mu_{f^{-1}\left[B_{i}\right]}(x) \\
& =\min _{i=1,2} \mu_{B_{i}} f(x) \\
& =\mu_{B_{1} \cap B_{2}} f(x) \\
& =\mu_{f^{-1}\left[B_{1} \cap B_{2}\right]}(x) .
\end{aligned}
$$

Similarly, it is not difficult to see that $\nu_{V_{1} \cap V_{2}}=$ $v_{f^{-1}\left[B_{1} \cap B_{2}\right]}$. Hence, we get $V_{1} \cap V_{2} \in \tau^{*}$.

(T3) Assume that $\left\{V_{i}\right\}_{i \in I}$ be an arbitrary sub-family of $\tau^{*}$. Then for any $i \in I$ there exists $B_{i} \in \tau$ such that $f^{-1}\left[B_{i}\right]=V_{i}$ which implies $\mu_{f^{-1}\left[B_{i}\right]}=\mu_{V_{i}}$ and $v_{f^{-1}\left[B_{i}\right]}=v_{V_{i}}$. Therefore, one can get for any $x \in X$ that

$$
\begin{aligned}
& \mu \bigcup_{i \in I} V_{i}(x)=\sup _{i \in I} \mu_{V_{i}}(x) \\
&=\sup _{i \in I} \mu_{f^{-1}\left[B_{i}\right]}(x) \\
&=\sup _{i \in I} \mu_{B_{i}} f(x) \\
&=\mu \bigcup_{i \in I} B_{i} f(x) \\
&=\mu \\
& f^{-1}\left[\bigcup_{i \in I} B_{i}\right]^{(x) .}
\end{aligned}
$$

On the other hand, it is easy to see that $\bigcup_{i \in I} V_{i}=$ $v_{f^{-1}}\left[\bigcup_{i \in I} B_{i}\right]$. Thus, we have $\bigcup_{i \in I} V_{i} \in \tau^{*}$.

From Theorem 2, the continuity of $f$ is trivial. Now, we prove that $\tau^{*}$ is the coarsest Pythagorean fuzzy topology over $X$ such that $f$ is Pythagorean fuzzy continuous. Let $\tau^{* *} \subset \tau^{*}$ be a Pythagorean fuzzy topology over $X$ such that $f$ is Pythagorean fuzzy continuous. If $B \in \tau^{*}$ then there exists $V \in \tau$ such that $f^{-1}[V]=B$. Since, $f$ is Pythagorean fuzzy continuous with respect to $\tau^{* *}$ we have $B=f^{-1}[V] \in \tau^{* *}$. Hence, we have $\tau^{* *}=\tau^{*}$.

\section{Conclusion}

In this paper, first of all, we introduce the notion of Pythagorean fuzzy topological space which extends the notions of both fuzzy topological space and intuitionistic fuzzy topological space. Then, we define the image and preimage of Pythagorean fuzzy subsets of a function's domain and co-domain, respectively. We also obtain some properties of these notions. Using the notions of image and pre-image, we introduce the Pythagorean fuzzy continuity of a function defined between two Pythagorean fuzzy topological spaces and we characterize Pythagorean fuzzy continuity of functions. We also prove that one can obtain a Pythagorean fuzzy topological space on a non-empty set using the concept of Pythagorean fuzzy continuity.

Acknowledgements We gratefully thank the referees for their valuable comments and suggestions which substantially helped improving the quality of the paper.

Open Access This article is distributed under the terms of the Creative Commons Attribution 4.0 International License (http://creativecomm ons.org/licenses/by/4.0/), which permits unrestricted use, distribution, and reproduction in any medium, provided you give appropriate credit to the original author(s) and the source, provide a link to the Creative Commons license, and indicate if changes were made.

\section{References}

1. Bourbaki N (1995) General Topology. Springer, Berlin, Heidelberg

2. Sardiu ME, Gilmore JM, Groppe B, Florens L, Washburn MP (2017) Identification of topological network modules in perturbed protein interaction networks. Sci Rep 7:43845

3. Lum PY, Singh G, Lehman A, Ishkanov T, Vejdemo-Johansson M, Alagappan M, Carlsson J, Carlsson G (2013) Extracting insights from the shape of complex data using topology. Sci Rep 3:1236

4. Nicolau M, Levine AJ, Carlsson G (2011) Topology based data analysis identifies a subgroup of breast cancers with a unique mutational profile and excellent survival. Proc Natl Acad Sci 108:72657270

5. Li L, Cheng WY, Glicksberg BS, Gottesman O, Tamler R, Chen R, Bottinger EP, Dudley JT (2015) Identification of type 2 diabetes subgroups through topological analysis of patient similarity. Sci. Transl. Med. 7:311ra174

6. Hofer C, Kwitt R, Niethammer M, Uhl A (2017) Deep learning with topological signatures. Adv Neural Inf Process Syst 30:1634-1644

7. Witten E (1996) Reflections on the fate of spacetime. Phys Today 96(4):2430

8. Chang CL (1968) Fuzzy topological spaces. J Math Anal Appl 24(1):182-190 
9. Lowen R (1976) Fuzzy topological spaces and fuzzy compactness. J Math Anal Appl 56(3):621-633

10. Lowen R (1977) Initial and final fuzzy topologies and the fuzzy Tychonoff theorem. J Math Anal Appl 58(1):11-21

11. Coker D (1997) An introduction to intuitionistic fuzzy topological spaces. Fuzzy Sets Syst 88(1):81-89

12. Coker D, Haydar Es A (1995) On fuzzy compactness in intuitionistic fuzzy topological spaces. J Fuzzy Math 3:899-910

13. Turanli N, Coker D (2000) Fuzzy connectedness in intuitionistic fuzzy topological spaces. Fuzzy Sets Syst 116(3):369-375

14. Ozcag S, Coker D (1998) On connectedness in intuitionistic fuzzy special topological spaces. Int J Math Math Sci 21(1):33-40

15. Kramosil I, Michálek J (1975) Fuzzy metrics and statistical metric spaces. Kybernetika 11(5):336-344

16. Riaz M, Hashmi MR (2018) Fuzzy parameterized fuzzy soft compact spaces with decision-making. Punjab UJ Math 50(2):131-145

17. Riaz M, Hashmi MR (2017) Fuzzy parameterized fuzzy soft topology with applications. Ann Fuzzy Math Inf 13(5):593-613

18. Zadeh LA (1965) Fuzzy sets. Inf Control 8(3):338-353

19. Bellman RE, Zadeh LA (1970) Decision-making in a fuzzy environment. Manag Sci 17(4):B-141

20. Atanassov KT (1986) Intuitionistic fuzzy sets. Fuzzy Sets Syst 20(1):87-96

21. Karnik NN, Mendel JM (2001) Operations on type-2 fuzzy sets. Fuzzy Sets Syst 122(2):327-348

22. Liang Q, Mendel JM (2000) Interval type-2 fuzzy logic systems: theory and design. IEEE Trans Fuzzy Syst 8(5):535-550

23. Yager RR (2013) Pythagorean fuzzy subsets. In: 2013 joint IFSA world congress and NAFIPS annual meeting (IFSA/NAFIPS), IEEE, Edmonton, AB, Canada, 24-28 June 2013, pp 57-61. https:// doi.org/10.1109/IFSA-NAFIPS.2013.6608375

24. Yager RR, Abbasov AM (2013) Pythagorean membership grades, complex numbers, and decision making. Int J Intell Syst 28(5):436452

25. Yager RR (2014) Pythagorean membership grades in multicriteria decision making. IEEE Trans Fuzzy Syst 22(4):958-965

26. Garg H (2018) Linguistic Pythagorean fuzzy sets and its applications in multiattribute decision-making process. Int J Intell Syst 33(6): 1234-1263

27. Garg H (2018) Hesitant Pythagorean fuzzy sets and their aggregation operators in multiple-attribute decision-making. Int J Uncertain Quantif 8(3):267-289

28. Garg H (2017) A novel improved accuracy function for interval valued pythagorean fuzzy sets and its applications in the decisionmaking process. Int J Intell Syst 32(12):1247-1260
29. Wei G, Garg H, Gao H, Wei C (2018) Interval-valued pythagorean fuzzy Maclaurin symmetric mean operators in multiple attribute decision making. IEEE Access 6:67866-67884

30. Garg H (2019) New logarithmic operational laws and their aggregation operators for Pythagorean fuzzy set and their applications. Int J Intell Syst 34(1):82-106

31. Garg H (2018) Generalised Pythagorean fuzzy geometric interactive aggregation operators using Einstein operations and their application to decision making. J Exp Theor Artif Intell 30:1-32

32. Garg H (2018) New exponential operational laws and their aggregation operators for intervalvalued pythagorean fuzzy multicriteria decision-making. Int J Intell Syst 33(3):653-683

33. Garg H (2018) Hesitant Pythagorean fuzzy Maclaurin symmetric mean operators and its applications to multiattribute decisionmaking process. J Intell Syst Int 1:1. https://doi.org/10.1002/int.22067

34. Garg H (2017) Confidence levels based Pythagorean fuzzy aggregation operators and its application to decision-making process. Comput Math Organ Theory 23(4):546-571

35. Garg H (2017) Generalized Pythagorean fuzzy geometric aggregation operators using Einstein tnorm and tconorm for multicriteria decision making process. Int J Intell Syst 32(6):597-630

36. Garg H (2016) A new generalized Pythagorean fuzzy information aggregation using Einstein operations and its application to decision making. Int J Intell Syst 31(9):886-920

37. Peng X, Selvachandran G (2017) Pythagorean fuzzy set: state of the art and future directions. Artif Intell Rev 2017:1-55

38. Peng X, Yuan H, Yang Y (2017) Pythagorean fuzzy information measures and their applications. Int J Intell Syst 32(10):991-1029

39. Peng X, Yang Y (2016) Pythagorean fuzzy choquet integral based MABAC method for multiple attribute group decision making. Int J Intell Syst 31(10):989-1020

40. Peng X, Yang Y (2015) Some results for Pythagorean fuzzy sets. Int J Intell Syst 30(11):1133-1160

Publisher's Note Springer Nature remains neutral with regard to jurisdictional claims in published maps and institutional affiliations. 